\title{
Comparative judgments of distal size: A chronometric analysis
}

\author{
RICHARD PRINGLE \\ Goucher College, Towson, Maryland \\ and \\ JOHN UHLARIK \\ Kansas State University, Manhattan, Kansas
}

\begin{abstract}
Observers were required to make comparative judgments of the distal sizes of squares at various perceived distances in a pictorial array. It was predicted that observers would normalize distance prior to judgment. Chronometric analyses indicated that the time to make "same" judgments increased systematically with the relative distance of the two stimuli. The time required to make "different" judgments depended on the nature of the difference. When the stimuli differed in proximal size, distal size, and distance, response time increased with distance ratio. However, when the stimuli differed in distal size and distance but not proximal size, response time decreased with distance ratio. In addition, when the stimuli differed in both distal and proximal size but not distance, RT decreased with size ratio. These results are consistent with a class of models that incorporate distance normalization into the comparative size-judging process. These and alternative models are discussed.
\end{abstract}

Using pictorial perspective arrays, Uhlarik, Pringle, Jordan, and Misceo (1980) had observers make magnitude estimates of distal and proximal size under a variety of instructional conditions. The time required to scale distal size varied directly with the distal size ratio of the comparison stimulus to the standard stimulus, but was independent of both the perceived distance of the comparison and the perceived distance separating the comparison from the standard. In addition, the response time for distal size scaling was unaffected by variation of proximal size. These results, along with introspective reports, indicated that distal size, as defined by pictorial cues, was scaled by counting or subitizing the number of standard units subtended by the comparison. This sort of sizescaling process is similar to the linear measurement process involved in the psychophysics of line length (e.g., Hartley, 1977, 1981). In our case, however, the "standard unit" was defined by linear perspective and textural detail so that the scale unit remained a constant distal size throughout the array. The results for retinal instructional conditions indicated that proximal size was also scaled by this sort of counting strategy. However, the nature of the scale unit dif-

A version of this paper was presented at the annual meeting of the Psychonomic Society, Phoenix, Arizona, 1979. Requests for reprints should be sent to Richard Pringle, Department of Psychology, Goucher College, Towson, Maryland 21204. We wish to thank Kevin Jordan and Giovanni Misceo for their help in preparing the stimuli used in this study. fered from the one used for distal size scaling. Specifically, for proximal size scaling the observers attempted to define the standard unit independently of the perspective array.

The lack of an effect of distance between the standard and the comparison on the time required to scale distal size suggests that repeated use of the same standard allowed the observer to maintain a mental representation of the standard unit throughout the textured array. Hence, the observer could apprehend the number of such units subtended by the comparison, regardless of position of the comparison in the array. These results are consistent with the notion that the perception of distal size in pictorial arrays is based on properties of scale (see Gibson, 1950, 1966). In other words, the textural and perspective detail of the pictorial array provides a referent scale unit of constant distal size that serves as the ruler for an explicit size measurement process. There were no indications that absolute distance and proximal size information entered into the size-scaling process.

The present study extends this analysis to comparative judgments of distal size, as defined by pictorial cues, by examining the manner in which variations of size and distance affect the time required to make "same-different" judgments of distal size. Two possibilities are suggested by the results of Uhlarik et al. (1980). One possibility is that observers separately scale the two sizes relative to a perceptual unit and then simply compare outputs. This strategy predicts no effect of distance on reaction time, but, instead, 
predicts that reaction time will depend primarily on the distal sizes of the two stimuli. The other possibility is that the observer compares the two stimuli directly. According to this strategy, the "measuring unit" would be the scale transection made by one of the stimuli, and the process would terminate when the second stimulus was assessed to have transected an equal or unequal portion of the perspective array. Because the referent stimulus would vary on each trial, the observer would be unable to preselect a standard distal unit to use throughout the array. Hence, on each trial, prior to making the size comparison, the observer would have to project the scale transection made by one stimulus into the same depth as the other stimulus. This projection would take time and should produce a reaction-time function that increases with the relative distance of the two stimuli. That is, distance would have to be normalized before comparative judgments of distal size could be made. This transformation is conceived here as being similar to the analogue processes required to make comparative shape judgments when the relative stimuli differ in orientation and/or size (cf. Bundesen \& Larsen, 1975; Cooper \& Shepard, 1978; Dixon \& Just, 1978).

The principal characteristic of both the measurement and the normalization strategies is that they rely directly on the properties of the scale implicit in the perspective array. We expect no evidence that observers somehow assess and integrate absolute proximal size and absolute distance information in order to compute distal sizes algorithmically and compare them. The assessment of proximal size, relative to that of distal size, takes considerable time and effort (Uhlarik et al., 1980), and reliance on absolute proximal size in the present study would produce systematic effects on reaction time.

\section{METHOD}

\section{Subjects}

Eight introductory psychology students served as subjects for course credit. Normal visual acuity, or vision corrected to at worst $20 / 30$, was a prerequisite for service in the experiment.

\section{Stimuli}

It has been demonstrated that relatively accurate distance perception is maintained for both photographs and perspective drawings of three-dimensional scenes (e.g., Hagen, 1978; Hagen \& Jones, 1978; Smith \& Gruber, 1958; Smith, Smith, \& Hubbard, 1958). Furthermore, Uhlarik et al. (1980; cf. Gibson, 1971) have provided evidence suggesting that the same size-scaling processes underlying constancy evoked by real three-dimensional scenes are present in the perception of two-dimensional arrays. In an attempt to eliminate systematic reaction-time effects due to accommodation and vergence eye movements necessary to fixate objects at different distances, the viewing conditions in the present study involved only pictorial cues for depth that could be displayed in a single depth plane. Figure 1 shows an achromatic example of the photographic stimulus array, which consisted of red cardboard squares on a receding surface.

The stimulus array was photographed with a $35-\mathrm{mm}$ single-lens reflex camera with a $50-\mathrm{mm} / 1.4$ macrolens. The camera was mounted with the lens $.7 \mathrm{~m}$ above the textured array. Slides were made from Kodak high-speed Ektachrome (E6) film with a lens opening of $\mathrm{f} / 22$, which allowed the stimulus blocks to be adequately focused at all distances.

The three-dimensional array consisted of two $.92 \times 7.3 \mathrm{~m}$ panels of textured cloth joined by a visible seam. Each panel had a slight crease down its middle. The cloth was dark blue with white polka dots $6 \mathrm{~mm}$ in diameter and uniformly distributed at a density of $.6 / \mathrm{cm}^{2}$. The array was placed on the floor of an evenly illuminated room. Because the lighting came from directly above and extended uniformly throughout the array, no shadows were cast by the stimuli. The stimuli consisted of red cardboard squares of various sizes photographed at various distances from the camera.

Two squares were presented on any given trial, and the observer's task was to decide if the squares were of the same or different distal sizes. For purposes of experimental design, the square on the left side of the array was arbitrarily designated as the standard. It had a distal width of either 10 or $15 \mathrm{~cm}$ and was presented either 2 or $8 \mathrm{~m}$ from the camera (observer). The comparison square was always on the right side of the array but could be at any of five photographic distances $(2,3,4,6$, or $8 \mathrm{~m})$. The comparisons varied in size as described below.

There were 20 stimulus pairs in which the standard and the comparison had the same distal sizes, with the comparison occurring at each of the five distances; the first comparison-size column of Table 1 summarizes these "same" stimuli. There were 40 stimulus pairs in which the standard and the comparison had different distal sizes, with the comparison occurring at each of the five distances; the second comparison-size column of Table 1 summarizes these "different" stimuli.

In addition there were two sets of control stimuli. There were 16 stimulus pairs in which the standard and the comparison had different distal sizes but subtended the same visual angle, with the comparison occurring at each of the four distances other than the distance of the standard; the left side of Table 2 summarizes these control "different" stimuli. There were 16 stimulus pairs in which the standard and the comparison had different distal sizes, with the comparison occurring at the same distance as the standard; these control "different" stimuli are summarized in the right side of Table 2. Note that there are five stimuli listed in Table 1 that are also included among the control "different" stimuli in Table 2 because, for these stimuli, the standard and the comparison had equal proximal sizes or were presented at equal distances.

Some filler stimuli were also included, and these consisted of an additional set of 20 "same" stimuli involving the distal sizes 5 and $20 \mathrm{~cm}$. This additional set served two purposes. First, it allowed for the approximate equalization of the number of "same" and "different" stimuli in each session. Second, without it, observers could conceivably have based their judgments on the fact that all 5- and 20-cm distal size stimuli were "different"; the fillers served to decrease the utility of this extraneous cue. These stimuli were considered fillers, not experimental stimuli, because no "different" stimuli having 5 - or $20-\mathrm{cm}$ standards were included.

\section{Procedure}

Stimuli were presented by a random-access slide projector in conjunction with a rear-projection screen $1.9 \mathrm{~m}$ from the lens of the projector and $.76 \mathrm{~m}$ from the observer. The projected field was $.46 \mathrm{~m}$ high and $.32 \mathrm{~m}$ wide. The visual angle subtended by the blocks as projected on the screen duplicated those that would have been subtended if an observer were substituted for the camera in the three-dimensional photographic field. Furthermore, the horizon line (as defined by the linear perspective) in the pictorial array was set at the observer's eye level. The projection screen was viewed binocularly; the observer's viewing position was restricted with a head- and chinrest. These procedures were designed to duplicate the three-dimensional scene as closely as possible.

Observers were instructed that each slide contained two red cardboard squares standing perpendicularly to the floor, and that their task was to decide if the two squares were of the same or 


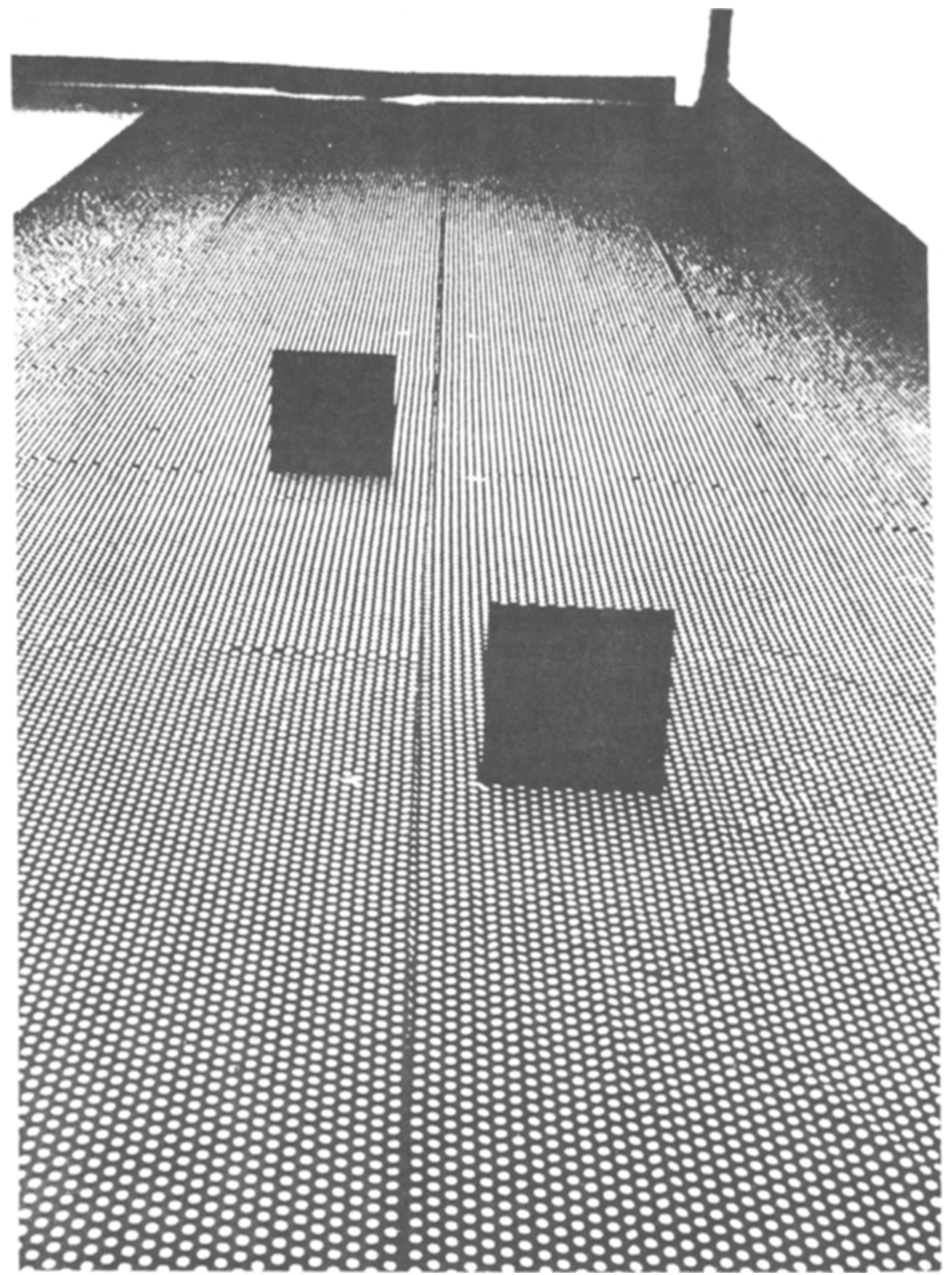

Figure 1. An achromatic example of the pictorial array with a "same" stimulus combination. The stimuli include the 15-cm square (standard) at $2 \mathrm{~m}$ and $15-\mathrm{cm}$ square (comparison) at $4 \mathrm{~m}$.

different sizes, regardless of position. They were told that, although the projected image of any square gets smaller with increasing distance, they should not be concerned with image size but should base their judgments on the actual or objective sizes of the squares placed on the receding three-dimensional surface.

Each observer was tested individually. The observer initiated a trial by pressing a switch that both started a timer and opened a shutter on the slide projector. Verbal "same" or "different" re- sponses activated an electronic voice key that both stopped the timer and closed the projection shutter. Feedback regarding whether a judgment was correct or incorrect in terms of the objective instructional set was provided after every trial. Trials on which errors occurred were repeated once, later in the same session. Prior to the initiation of each trial, the observer fixated a luminous 6-mm point on the projection screen. To control for any possible systematic differences in visual search time involved in 
Table 1

Standard and Comparison Combinations for "Same" and "Different" Stimuli

\begin{tabular}{|c|c|c|c|}
\hline \multicolumn{2}{|c|}{ Standard } & \multicolumn{2}{|c|}{ Comparison Size } \\
\hline Distance & Size & "Same" & "Different" \\
\hline 2 & 10 & 10 & 5,15 \\
\hline 2 & 15 & 15 & 10,20 \\
\hline 8 & 10 & 10 & 5,15 \\
\hline 8 & 15 & 15 & 10,20 \\
\hline
\end{tabular}

Note-Distance is given in meters; size is given in centimeters. Each comparison stimulus was presented at each of five photographic distances: $2,3,4,6$, and $8 \mathrm{~m}$.

scanning the array, half the observers fixated the dot at the middle of the top edge of the array, and the other half at the middle of the bottom edge. Each observer was tested in four sessions on different days. Different random orders of the stimuli were used for each session, which contained four replications of "same" stimuli, two replications of "different" stimuli, and two replications of the "same" filler stimuli. In a single session there were 120 "same" stimuli and 134 "different" stimuli. The first session was considered practice and was not used in the final data analysis. At the beginning of each session, subjects were presented 24 warmup trials.

\section{RESULTS}

Separate analyses of variance were performed for the "same" stimuli and the three types of "different" stimuli. These analyses were performed on "correct" RTs when available. That is, when the observers made an error, the trial was later repeated. If the response was correct the second time, that RT was used in the data analysis. However, when errors occurred on both the initial and repeated presentations of a stimulus, the first "incorrect" response time was included in the data anlysis. This occurred only among the experimental "different" stimuli (i.e., Table 1) and accounted for $2.2 \%$ of the data entries for that condition. Analyses based only on correct responses revealed results essentially equivalent to those discussed below.

\section{Same Stimuli}

The design for the "same" stimuli consisted of five factors: position of the fixation point (top or bottom), replications, size of the standard $(10$ or $15 \mathrm{~cm})$, distance of the standard stimulus from the camera ( 2 or $8 \mathrm{~m}$ ), and the distance ratio of the standard and the comparison (see Table 1). The overall error rate was $4.3 \%$ and generally increased with distance ratio. An analysis of variance of RT for the stimuli having distance ratios greater than 1.0 showed a significant main effect of distance ratio $[F(3,18)=19.21, \mathrm{p}<.01]$, shown in Figure 2. RT increased monotonically with relative distance, and, excluding the first data points (distance ratio $=1.0$ ), which represent the "same" stimuli having no distance disparity, the increasing functions are approximately linear. In addition to this main effect, the distal size of the standard interacted with distance disparity $[F(3,18)=6.88, p<.01]$. This interaction, shown graphically in Figure 3, reflects a difference in slope associated with the 10and $15-\mathrm{cm}$ standards. No other main effects or interactions were statistically significant.

The "same" stimuli having a distance ratio of 1.0 represent the special case of identical stimuli presented side by side; they were not included in the analysis above but were analyzed separately. The only statistically significant effect associated with these stimuli was a main effect of position of the fixation point $[F(1,6)=20.36, p<.01]$. The top fixation led to overall faster response times than the bottom fixation.

An analysis of variance that included both sets of

Table 2

Stimulus Combinations for the Control "Different" Stimuli

\begin{tabular}{|c|c|c|c|c|c|c|c|c|c|}
\hline \multicolumn{2}{|c|}{ Standard } & \multicolumn{8}{|c|}{ Comparison } \\
\hline \multirow[t]{3}{*}{ Distance } & \multirow[t]{3}{*}{ Size } & \multicolumn{4}{|c|}{ Variation of Distance } & \multicolumn{4}{|c|}{ Variation of Proximal Size } \\
\hline & & \multicolumn{4}{|c|}{ Distance Ratio (Comparison/Standard) } & \multicolumn{4}{|c|}{ Proximal Size Ratio (Standard/Comparison) } \\
\hline & & $\begin{array}{l}1.5 \\
(3)\end{array}$ & $\begin{array}{l}2.0 \\
\text { (4) }\end{array}$ & $\begin{array}{l}3.0 \\
(6)\end{array}$ & $\begin{array}{l}4.0 \\
(8)\end{array}$ & $\begin{array}{l}1.5 \\
(2)\end{array}$ & $\begin{array}{l}2.0 \\
(2) \\
\end{array}$ & $\begin{array}{l}3.0 \\
\text { (2) }\end{array}$ & $\begin{array}{l}4.0 \\
\text { (2) }\end{array}$ \\
\hline \multirow{4}{*}{$\begin{array}{l}2 \\
2 \\
\end{array}$} & 10 & 15 & 20 & 30 & 40 & 6.7 & 5.0 & 3.3 & 2.5 \\
\hline & 15 & 27.5 & 30 & 45 & 60 & 10 & 7.5 & 5.0 & 3.8 \\
\hline & & \multicolumn{4}{|c|}{ Distance Ratio (Standard/Comparison) } & \multicolumn{4}{|c|}{ Proximal Size Ratio (Standard/Comparison) } \\
\hline & & $\begin{array}{l}1.3 \\
(6) \\
\end{array}$ & $\begin{array}{l}2.0 \\
\mathbf{( 4 )} \\
\end{array}$ & $\begin{array}{l}2.7 \\
\text { (3) }\end{array}$ & $\begin{array}{l}4.0 \\
(2) \\
\end{array}$ & $\begin{array}{r}1.3 \\
(8) \\
\end{array}$ & $\begin{array}{l}2.0 \\
(8) \\
\end{array}$ & $\begin{array}{l}2.7 \\
(8)\end{array}$ & $\begin{array}{l}4.0 \\
\text { (8) }\end{array}$ \\
\hline 8 & 10 & 7.5 & 5.0 & 3.3 & 2.5 & 13.3 & 20 & 26.7 & 40 \\
\hline 8 & 15 & 11.2 & 7.5 & 5.6 & 3.7 & 20 & 30 & 40 & 60 \\
\hline
\end{tabular}

Note-Distance is given in meters; size is given in centimeters. Entries represent the distal size of the comparison stimulus. The distance of the comparison (from camera) is indicated in parentheses at the top of each column. 


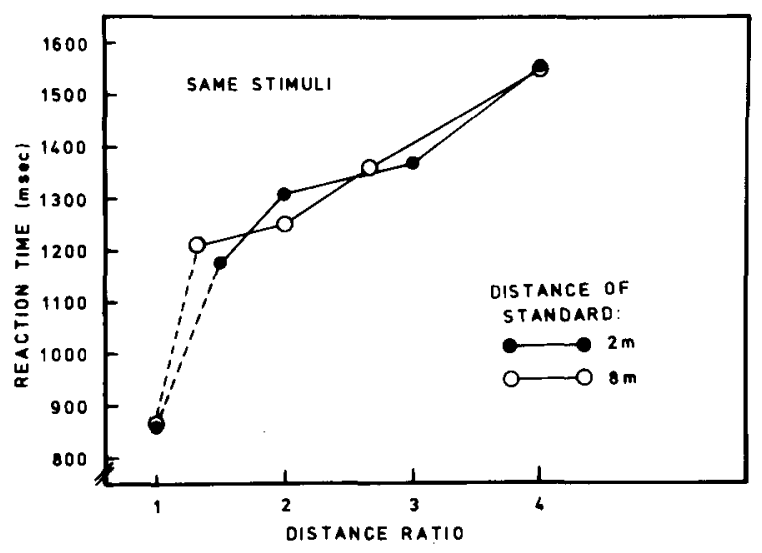

Figure 2. Mean reaction time as a function of distance ratio for "same" stimuli. Dotted lines connect points having zero distance disparity (distance ratio $=1.0$ ).

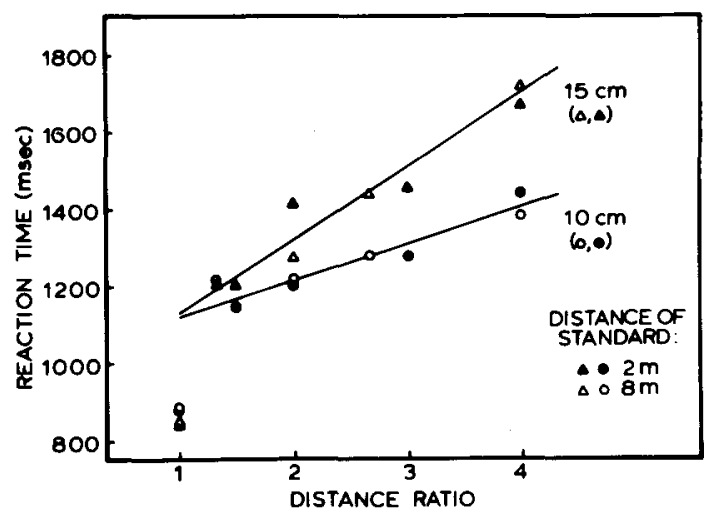

Figure 3. Mean reaction time as a function of distance ratio for "same" stimuli shown for both the $10-\mathrm{cm}$ and $15-\mathrm{cm}$ stimuli.

"same" stimuli revealed results identical to those reported here for the "same" stimuli involving distance ratios greater than 1.0 .

\section{Different Stimuli}

The statistical design for the "different" stimuli (Table 1) was similar to the design for the "same" stimuli. However, there was one additional factor because the comparison stimulus could be either smaller or larger in distal size than the standard. The effect of distance ratio on RT for the "different" stimuli is shown in Figure 4 and is similar to the data shown for "same"' stimuli in Figure 2. Overall, these "different" stimuli led to a high error rate, $16.4 \%$, and these errors were clustered among stimuli with large distance disparities. For example, three subjects consistently judged the $15-\mathrm{cm}$ standard at $2 \mathrm{~m}$ to be equal to the $20-\mathrm{cm}$ comparison at $8 \mathrm{~m}$. Apparently, for some subjects, some of the distal size differences were not discriminable at the greater distance disparities. However, the striking similarities in the RT functions for the "same" and "dif- ferent" stimuli (compare Figures 2 and 4) suggest that, in spite of some subjects' difficulties in making correct discriminations for the greater distance ratios, they nevertheless utilized a consistent responsedecision strategy. Apparently, errors were, in general, not aberrations in the information-processing sequence but, rather, a failure of resolution. In support of this assertion, we note that the RT data for the three subjects mentioned above were similar to the functions shown in Figures 2, 3, and 4.

An analysis of variance on the "different"-stimuli RTs revealed that, overall, slower times were associated with the $15-\mathrm{cm}$ standard than with the $10-\mathrm{cm}$ standard $(1,231 \mathrm{msec}$ and $1,145 \mathrm{msec}$, respectively) $[F(1,6)=20.20, p<.01]$. In addition, there was a significant main effect of distance ratio $[F(4,24)=18.82$, $p<.01]$. As shown in Figure 4, reaction time increased with distance ratio. For these "different" stimuli, proximal size varied simultaneously with variations in distance. However, the variation of proximal size was not exactly reciprocal to the variation in distance. Consequently, the $\mathrm{F}$ ratio for distance ratio does not accurately reflect an impact of proximal size ratio. For the "same stimuli" (see Figure 2), proximal size and distance varied both concomitantly and reciprocally, and therefore it is not possible to determine which of the two dimensions is responsible for the increasing RT functions in Figures 2 and 3. For the "different" stimuli condition, however, these stimulus dimensions were not perfectly correlated. For example, a standard-comparison distance ratio of 2.0 could be associated with proximal size ratios ranging from 1.0 to 4.0 . When the data summarized in Figure 4 are partitioned and replotted as a function of proximal size ratio, different functions result. The data are noisy and, in fact, do not increase monotonically with proximal size ratio. A regression analysis indicated that only $24 \%$ of the variance could be accounted for by a linear relationship between proximal size ratio and reaction time. For the mean response times in Figure 4, on the other



Figure 4. Mean reaction time as a function of distance ratio for "different" stimuli. 
hand, linearity accounts for $85 \%$ of the variance. This finding suggests that distance rather than proximal size is the critical dimension and that proximal size ratio has little systematic effect on reaction time.

\section{Control Stimuli}

When the standard and comparison differed in size but not distance (Table 3, right side), there was an overall error rate of $0.0 \%$. An analysis of variance of RTs showed a main effect of size ratio $[F(3,18)$ $=23.25, \mathrm{p}<.01 \mathrm{]}$. As shown in Figure 5, RT decreased systematically with size ratio. All other main effects and interactions were not statistically significant. There was no impact of replication on the effect of size disparity. This result is important because, otherwise, the decreasing function may have simply been the result of observers' learning that unusually large or small sizes were invariably associated with the "different" response. That type of learned response would have produced a replication by size disparity interaction.

When the standard and the comparison differed in distance but not in proximal size (Table 3, left side), error rate was low $(.3 \%)$. An analysis of variance of RT indicated significant main effects of the size of the standard $(930$ and $890 \mathrm{msec}$ for the $10-$ and $15-\mathrm{cm}$ standards, respectively) $[F(1,6)=14.10, p<.01]$, the distance of the standard from the camera (854 and $966 \mathrm{msec}$ for the 2- and 8-m distances, respectively) $[F(1,6)=32.32, p<.01]$, and distance ratio $[F(3,18)$ $=26.06, p<.01]$. All other effects were statistically insignificant, and, again, there was no impact of replication on the effect of distance ratio. The effect of distance ratio is shown in Figure 6. When the proximal sizes of the standard and the comparison were equal, an increase in distance ratio was associated with a decrease in RT. However, pairwise comparisons indicated that the only RT decrement that was statistically significant was between distance ratios $1.3(1.5)$ to $2.0[F(1,18)=36.73, p<.01]$.

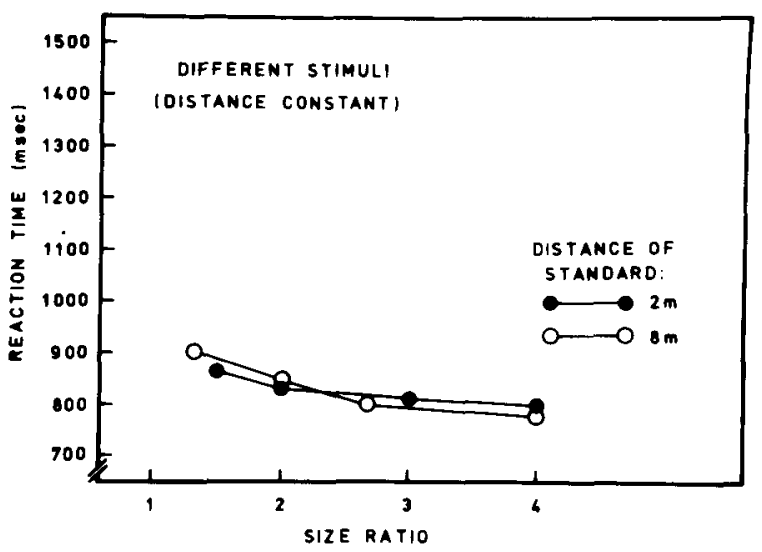

Figure 5. Mean reaction time as a function of proximal size ratio for the control "different" stimuli varying in size but not distance.



Figure 6. Mean reaction time as a function of distance ratio for the control "different" stimuli varying in distal size and distance but not proximal size.

In addition to these effects, there was a three-way interaction involving these same factors: size of standard, distance of standard, and distance ratio $[F(3,18)$ $=5.45, \mathrm{p}<.01]$. This interaction was due to slightly different rates of decline in RT as a function of distance ratio for the various sizes and positions of the standards. Finally, there was a main effect of fixation position $[F(1,6)=13.87, p<.01]$. Overall, the top fixation position led to faster response times than the bottom fixation did.

It should be noted that the results summarized in Figures 2-6 are inconsistent with the results obtained in similar experiments by Broota and Epstein (1973; Epstein \& Broota, 1975). In those studies, observers made comparative distal size judgments under threedimensional viewing conditions. It was reported that reaction time, regardless of stimulus class (e.g., "same," "different," "different" controls, etc.), varied as a function of the mean distance from observer to stimulus, and there were no systematic effects of the distance separating the stimuli. A difference between the studies that may be important is that the present study used only pictorial cues to depth in order to control for accommodation and vergence eye-movement time. If an observer's normal resting vergence and accommodation were set for distances closer than the stimulus array, then reaction time would be expected to increase with observer-stimulus separation simply as a result of the adjustments necessary to fixate objects at different distances. This peripheral effect on reaction time could have overshadowed an effect of relative distance, since Broota and Epstein used only a small range of distance ratios.

Scanning times between the standard and comparison stimuli represent a similar type of confounding in the present study. As the interstimulus distance increases, so does the scanning time required to sequentially fixate the two stimuli. Hence, one might expect RT to increase with distance disparity due to this factor alone. Two different fixation positions were included in an attempt to weigh the impact of 
scanning time. There were few effects of this factor on response time. There were no effects of fixation position on "same" stimuli or experimental "different" stimuli. The effects that occurred for the "same" stimuli that had zero distance disparity (distance ratio $=1.0$ ) and the control "different" stimuli were interpretable in terms of eye-movement patterns. However, the "scan times" involved were small (e.g., 35-70 msec) and cannot, in and of themselves, account for the increments of $700 \mathrm{msec}$ in Figures 2, 3 , and 4. Also, one would not expect the functions in Figures $2-4$ to be linear if they reflected eye scan. Indeed, an eye-scan interpretation would more likely predict functions that are curvilinear with distance ratio, because the vertical, projected separations among the five successive photographic distances decrease with distance due to foreshortening. Furthermore, these functions should be different for "topdown" vs. "bottom-up" fixation points and different for standards at 8 vs. $2 \mathrm{~m}$. For example, $7 \mathrm{deg}$ of visual angle on the viewing screen separated the stimuli presented at 2 and $4 \mathrm{~m}$, whereas approximately 1 deg separated stimuli presented at 6 and $8 \mathrm{~m}$. The RT functions, particularly those in Figure 2 , are nearly linear, and fixation point did not interact with position of standard and distance ratio. In addition, this scan-time explanation would predict increasing RT functions for the "different" stimulus control condition in which distance but not proximal size varied (Figure 6), but the experimental result did not agree with the prediction. Furthermore, it would be difficult to account for the interaction involving size of standard and distance ratio (Figure 3 ) with a scantime explanation.

Data on saccadic eye movements also make a scantime explanation of our results unlikely. The latency to make lateral saccadic eye movements is on the order of 150-200 msec (Saslow, 1967a, 1967b), but the difference in the total time required to make saccades to displacements of 8 vs. 16 deg is only a few milliseconds (Saslow, 1967b). Our observers were required to make a saccade on every trial, because a displacement of $15 \mathrm{deg}$ separated the bottom fixation point and the nearest stimulus (presented at $2 \mathrm{~m}$ ), and $7 \mathrm{deg}$ separated the top fixation point and the nearest stimulus (presented at $8 \mathrm{~m}$ ). On the other hand, only $13 \mathrm{deg}$ separated stimuli presented at the closest $(2 \mathrm{~m})$ and farthest $(8 \mathrm{~m})$ distance. Insofar as the latency to make the movement seems to be the most time-consuming and, once initiated, saccades are very fast (up to a peak velocity of $830 \mathrm{deg} / \mathrm{sec}$; Alpern, 1971), it seems unlikely that differences in vertical displacement of the stimuli on the viewing screen could account for the observed changes in RT as a function of distance ratio.

\section{DISCUSSION}

For the "same" stimuli and the experimental "dif- ferent" stimuli, RT increased as a function of the relative distance from the observer to the standard and the comparison squares. The absolute distance of the stimuli from the observer (camera) had little effect as indicated by the nearly overlapping functions in Figures 2 and 4 . That is, whether the standard was 2 or $8 \mathrm{~m}$ from the observer, RT increased systematically with relative distance, and, furthermore, there was no slope difference between the two functions. These results are consistent with a transformation process that normalizes the stimuli with respect to distance. The greater the distance ratio, the more time is required to complete normalization. Ignoring the special case in which the standard and the comparison are identical (distance ratio $=1.0$ ), the RT functions are approximately linear with the relative depth implicit in the pictorial array, not with the vertical distances actually separating the stimuli on the projection screen. Thus, the "distance normalization" cannot be simply a translation on the picture plane; more likely, it is a movement in perceived depth. The discontinuity in the RT functions that occurs between distance ratios of 1.0 and 1.3 / 1.5 presumably reflects the time required to invoke normalization processes. No normalization would be required for distance ratios of 1.0. These interpretations of the data are consistent with introspective reports: Subjects reported mentally projecting one stimulus through depth until it was adjacent to the other stimulus. Presumably, only at that point was the distal size comparison made. In other words, in order to make comparative judgments of distal size, one stimulus or its size equivalent (e.g., a projected scale transection) must be mentally moved through depth until it is in the same depth plane as the other stimulus.

Although distance would be the normalized dimension, proximal size would also have to be adjusted as the stimulus is moved through depth. Otherwise, the distal size of the stimulus would not be preserved. However, this proximal size adjustment might be accomplished indirectly by "zooming" the stimulus according to perspective cues in the array. Hence, we do not suggest that the observer need actively coordinate this proximal size transformation with movement in depth. Rather, the perspective cues inherent in the stimulus array provide the scale whereby the proximal size-distance zooming might be accomplished. That is, the observer would move or follow a scale transection from standard to comparison, and that process would, ipso facto, result in the proximal size zooming. One could argue that the presence of the scale would obviate the need for the normalization process; a more straightforward strategy would involve measuring one stimulus with reference to the scale, measuring the other stimulus in the same manner, and then comparing outputs. Presumably, such a process would lead to RT functions independent of distance and proximal size. In that case, the 
critical variable for $\mathrm{RT}$ would be the number of units subtended by the figures, that is, their distal sizes. However, such measures take considerable time (e.g., 1,500 to 2,500 msec; see Uhlarik et al., 1980), and performing two such measures prior to comparison would require response times much longer than those obtained in this study. Thus, it seems more likely that subjects directly compare scale transections made by the two stimuli, that is, that they project one stimulus or its transection into the same depth plane as the other to make the match. The perspective elements of the array could serve to funnel one stimulus through depth. The larger the stimulus, the more elements involved in the definition of the funnel, and that additional load could conceivably slow the rate of normalization. A slower rate of normalization for larger stimuli would be the basis of the interaction between the size of the standard and the distance disparity among the "same" stimuli shown graphically in Figure 3. That stimulus parameters sometimes affect normalization rate has been reported in the context of mental rotation (see, e.g., Corballis \& Roldan, 1975; Hochberg \& Gellman, 1977; Pringle \& Cowan, 1978). Presumably, such effects are due to an additional "load" arising from certain types of stimulus complexity on the mental transformational process. Variation of size in the present context may exert a similar effect.

The normalization model is supported by the decreasing RT function obtained for the control "different" stimuli involving the standard and the comparison stimuli presented at equal distances. Since there is no need to normalize distance when only size varies, discrimination RT functions should result (see Johnson, 1939; Snodgrass, 1975). That is, for the larger size differences, the differences are more apparent, and consequently more quickly detected. This type of decreasing RT function was also found for the "different" control stimuli whose distal size and distance were different from, but proximal size equal to, those of the standard. As distance disparity increases, thereby increasing the distal size disparity, the discrimination becomes easier rather than more difficult. In the normalization model presented above, distance must be normalized whenever the standard and comparison differ with respect to distance. The data shown in Figure 6 suggest that the model fails in this case. This figure plots RT as a function of distance ratio when the proximal sizes of the standard and comparison are equivalent. If distance always had to be normalized, Figure 6 should show an increasing RT function similar to the functions obtained in Figures 2, 3, and 4. Instead, RT initially decreases and then levels off with distance ratio. To hold proximal size constant in this condition, the distal size ratio had to increase in proportion to distance ratio. Perhaps normalization is required to compare nearly equal distal sizes, but may not be required when distal sizes are grossly differ- ent. Similar conditional normalization models have been proposed to account for number comparison processes (e.g., Dixon, 1978; Pringle, 1979, Note 1). Another possible explanation is that normalization is required for the stimuli in question, but, following completion of normalization, the time to complete size judgment generally decreases with size ratio. Then each successive increment in the distal size ratio would be associated with a normalization function having a lower y-intercept. Overall, the RT function in Figure 6 could result.

A third approach based upon a somewhat algorithmic account of distal size perception may be equally capable of accounting for the data. Suppose the observer used the following decision strategy to make comparative distal size judgments: (1) If the proximal sizes and the distances of the two squares are both equal, then the distal sizes must be equal; (2) if either proximal sizes or distances are equal, but not both, then the distal sizes must be unequal; (3) if proximal sizes and distances are unequal, then one cannot determine whether the distal sizes are equal without further processing. Normalization of distance would certainly be a candidate process in the latter case. Once distance (or proximal size, for that matter) was normalized, the task would reduce to case 1 or case 2 . Since cases 1 and 2 are based upon discrimination alone, one would expect decreasing RT functions similar to those obtained in Figures 5 and 6 . When both the proximal sizes and the distances differ, on the other hand, one would expect increasing RT functions characteristic of normalization, such as those found in Figures 2, 3, and 4.

Although there is insufficient evidence to allow a choice among these models, each proposes normalization of distance to account for the RT functions in Figures 2, 3, and 4. A nonnormalization model could be based on the observation that variability of size and distance ratio judgments increases with the magnitude of the relevant ratio (Baird, 1970). Variability can be considered an index of uncertainty; hence, RT to scale size or distance ratios ought to increase with the stimulus ratio. Suppose the observer scaled the proximal size ratio of the standard and comparison, scaled the distance ratio, and then compared the results. A "same" response would occur when the ratios were perceived equivalent (i.e., reciprocals). Regardless of whether the proximal size and distance ratios were established in series or in parallel, an RT function that increased with distance ratio would result. This approach could, in principle, account for case 3 . It combined with the algorithmic approach to cases 1 and 2 described above, the nonnormalizing model can account for the principle qualitative aspects of the data.

This model has some difficulty in accounting for the near linearity of the increasing RT functions and accounting for the slope differences for the 10- and $15-\mathrm{cm}$ standards in Figure 3. Why, assuming RT is 
a linear function of uncertainty, should uncertainty increase faster with size and distance ratios of a $15-\mathrm{cm}$ standard than a $10-\mathrm{cm}$ standard? A slightly different approach is to assume that the increasing RT functions are the direct result of the scaling processes themselves rather than the result of increasing levels of uncertainty. The ratio scaling of proximal size is known to produce an increasing RT function, and the data suggest the increment is due to "laying-off" processes (see Uhlarik et al., 1980); perhaps the ratio scaling of distance is based on a similar strategy. The completion of both scaling processes in series or in parallel would then produce the requisite RT function. This model could account for the near-linearity of the RT functions. Furthermore, the interaction in Figure 3 is very similar to an effect of the size of the standard stimulus on the "laying-off" RT functions in judgments of area and numerosity (Pringle \& Nagel, Note 2). One difficulty with both of these scaling models is that several studies have shown distal and proximal size scaling to be a rather slow process relative to the RT data obtained in the present study (see Hartley, 1981; Uhlarik et al., 1980). Assuming that distance ratio scaling has a similar time course, and assuming that the two scaling processes occur in series, then the resultant RT function would have little resemblance to those shown in Figures 2, 3, and 4.

In any case, the present study has taken an information-processing approach to comparative judgment of distal size, and the results indicate that normalization of distance may be an integral part of that process. In whatever manner the distance normalization model is refined to accommodate the results in Figure 6, the properties of scale, as defined by the perspective and textural details of the pictorial array, are conceived here as the basis for the distance transformation. Whether the stimulus itself or merely its scale transection is funneled through depth, the properties of scale would provide the grammar to allow preservation of the distal size of the stimulus throughout the displacement process. Thus, the present study proposes related models of how distal size scaling, based solely on properties of scale, might be accomplished.

\section{REFERENCE NOTES}

1. Pringle, R. Development of the normalization strategy in comparative numerosity judgments. Paper presented at the annual meetings of the Psychonomic Society, St. Louis, 1980.

2. Pringle, R., \& Nagel, J. A. The role of the standard in magnitude estimations of numerousness: Evidence for normalization and "laying-off" processes. Paper presented at the Eastern Psychological Association Meetings, Baltimore, 1982.

\section{REFERENCES}

ALPERN, M. Effector mechanisms in vision. In J. W. Kling \& L. Riggs (Eds.), Experimental psychology (3rd edition). New York: Holt, Rinehart \& Winston, 1971.
BAIRD, J. C. The psychophysical analysis of visual space. New York: Pergamon Press, 1970.

Bundesen, C., \& LARsen, A. Visual transformation of size. Journal of Experimental Psychology: Human Perception and Performance, 1975, 1, 214-220.

Broota, K. D., \& EPSTEin, W. The time it takes to make veridical size and distance judgments. Perception \& Psychophysics, 1973, 14, 358-364.

Cooper, L. A., \& Shepand, R. N. Transformations in representations of objects in space. In E. C. Carterette \& M. P. Friedman (Eds.), Handbook of perception (Vol. 8): Perceptual coding. New York: Academic Press, 1978.

Corballis, M. C., \& Roldan, C. E. Detection of symmetry as a function of angular orientation. Journal of Experimental Psychology: Human Perception and Performance, 1975, 1, 221-230.

Dixon, P. Numerical comparison processes. Memory \& Cognition, $1978,6,454-461$.

Dixon, P., \& JUst, M. A. Normalization of irrelevant dimensions in stimulus comparisons. Journal of Experimental Psychology: Human Perception and Performance, 1978, 4, 36-46.

Epste in, W., \& Broota, K. D. Attitude of judgment and reaction time in estimation of size at a distance. Perception \& Psychophysics, 1975, 18, 201-204.

GiBson, J. J. The perception of the visual world. Boston: Houghton Mifflin, 1950.

GiBson, J. J. The senses considered as perceptual systems. New York: Houghton Mifflin, 1966.

Gibson, J. J. The information available in pictures. Leonardo, 1971, 4, 27-35.

HAGEN, M. A. An outline of an investigation into the spatial character of pictures. In H. L. Pick \& J. E. Saltzman (Eds.), Modes of perceiving and processing information. Hillsdale, N.J: Erlbaum, 1978.

Hagen, M. A., \& Jones, R. L. Cultural effects on pictorial perception: How many words is one picture really worth? In R. D. Walk \& H. L. Pick (Eds.), Perception and experience (Vol. 6). New York: Plenum Press, 1978.

HARTLEY, A. A. Mental measurement in the magnitude estimation of length. Journal of Experimental Psychology: Human Perception and Performance, 1977, 3, 622-628.

HartLey, A. A. Mental measurement of line length: The role of the standard. Journal of Experimental Psychology: Human Perception and Performance, 1981, 7, 309-317.

Hochberg, J., \& Gellman, L. The effect of landmark features on mental rotation times. Memory \& Cognition, 1977, 5, 23-26.

Johnson, D. M. Confidence and speed in the two-category judgment. Archives of Psychology (New York), 1939, 34, No. 241.

Pringle, R. Perceived number equivalence by adults and children: A normalization model of size-density coordination (Unpublished doctoral dissertation, Kansas State University, 1979).

Pringle, R., \& Cowan, T. M. Mental rotation of possible and impossible four-cornered toruses. Perception \& Psychophysics, $1978,24,84-92$.

Saslow, M. G. Effects of components of displacement-step stimuli upon latency for saccadic eye movement. Journal of the Optical Society of America, 1967, 57, 1024-1030. (a)

SAsLow, M. G. Latency for saccadic eye movement. Journal of the Optical Society of America, 1967, 57, 1030-1033. (b)

Sмiтh, O. W., \& Gruber, H. Perception of depth in photographs. Perceptual and Motor Skills, 1958, 8, 307-313.

Smith, O. W., Smith, T. C., \& Hubbard, D. Perceived distance as a function of the method of presenting perspective. American Journal of Psychology, 1958, 71, 662-675.

Snodgrass, J. G. Psychophysics. In B. Scharf (Ed.), Experimental sensory psychology. Glenview, Ill: Scott, Foresman, 1975.

Uhlarik, J., Pringle, R., Jordan, K., \& Misceo, G. Size scaling in two-dimensional pictorial arrays. Perception \& Psychophysics, 1980, 27, 60-70.

(Manuscript received September 23, 1981; revision accepted for publication April 28, 1982.) 